\title{
ELECTRON-POSITRON COLLIDERS AND OTHER ACCELERATOR TECHNOLOGIES
}

SLAC-PUB- -5955

DE93 004018

\author{
Robert H. Siemann* \\ Stanford Linear Accelerator Center, \\ Stanford University, Stanford, CA 94309
}

\begin{abstract}
The accelerator physics and technology of hadron colliders, heavy quark factories, and linear colliders are reviewed. The status and performance of major high energy accelerators are summarized.
\end{abstract}

\section{INTRODUCTION}

Accelerators and accelerator technology are among the most important factors determining the frontier of particle physics. This paper is a summary of a talk presented at the 1992 International Conference on High Energy Physics. It was a peculiar talk for two reasons. First, the SSC and LHC are the major high energy physics construction projects at the present time, and HERA has just been completed and is starting to operate. Each of these had a dedicated talk, and, therefore, these projects, but not associated accelerator physics and technology, were outside the scope of the talk.

Second, the talk was presented to an audience of particle physicists and does not have the technical detail that you would find at an accelerator conference. The hope is give an overview of the status of major accelerators and a perspective of accelerators and accelerator science to physicists whose work depends critically on that science but who are not directly involved in it.

* Work supported by the Department of Energy, contract DE-AC03-76SF00515.

\section{HADRON COLLIDERS}

The TEVATRON [1]

During the previous TEVATRON collider run in $1988-1989$ the typical peak luminosity was $1.6 \times 10^{30} \mathrm{~cm}^{-2} \mathrm{~s}^{-1}$ and the total integrated luminosity was $9.6 \mathrm{pb}^{-1}$. The long term goal is to reach $5 \times 10^{31} \mathrm{~cm}^{-2} \mathrm{~s}^{-1}$ with a concomitant increase in integrated luminosity. The luminosity,

$$
\varepsilon=\frac{1}{4 \pi} \frac{N_{1} N_{2} f_{C}}{B^{2} \sigma_{x} \sigma_{y}},
$$

can be rewritten in different ways to reflect different performance limits. (In this equation $\mathrm{N}_{1}$ and $\mathrm{N}_{2}$ are the total number of particles in each beam, $\mathrm{f}_{\mathrm{c}}$ is the collision frequency, $\mathrm{B}$ is the number of bunches, and $\sigma_{x}$ and $\sigma_{y}$ are the rms transverse sizes.) The TEVATRON limitations are the number of $\bar{p}$ 's and the phase space density, $N /(B \varepsilon)$ where $\varepsilon$ is the invariant emittance, of the proton beam. The former comes from the difficulty of producing $\overline{\mathrm{p}}$ 's and the latter from the tune spread of the proton beam at low energies due to space charge and the beam-beam effects experienced by the $\bar{p}$ 's during collisions. Taking account of these limits the luminosity becomes

P. intcd at the XXVI Internationai Conference on High Energy Physics (ICHEP 92) Dallas, Texas, August 6-12, 1992 


$$
\varepsilon=N_{p} \frac{N_{p}}{B \varepsilon_{p}} \frac{\gamma\left(f_{c} / B\right)}{\pi \beta^{*}\left(1+\varepsilon_{-p} / \varepsilon_{p}\right)} .
$$

$\gamma$ is the beam energy in units of $\mathrm{mc}^{2}$ and $\beta^{*}$ is the "beta function" that measures the depth-offield of the focus at the collision point. Note that $f_{c} / B$ is independent of the number of bunches.

The elements for reaching $5 \times 10^{31} \mathrm{~cm}^{-2} \mathrm{~s}^{-1}$ are: a) $\bar{p}$ source improvements, b) stronger focusing at the interaction points to reduce $\beta^{*}$, c) electrostatic separators to avoid unwanted collisions and thereby relax one of the limits on $\mathrm{N}_{\mathrm{p}} / \mathrm{B} \varepsilon_{\mathrm{p}}$ by reducing the beam-beam effect on the $\overline{\mathrm{p}}$ 's, $d$ ) a linac energy increase that reduces the space charge tune spread and relaxes the other limit on $\mathrm{N}_{\mathrm{p}} / \mathrm{B} \varepsilon_{\mathrm{p}}$, and e) the Main Injector that will allow further intensity increases. The first three of these are completed, and the expected benefits realized. The commissioning of and operating with electrostatic separators is especially important because it is key for realizing the benefits from the linac upgrade and the Main Injector. The linac upgrade is almost complete with commissioning scheduled to begin in the winter of 1993, and Main Injector beam is scheduled for early in 1997.

With these improvements the TEVATRON will be remain a centerpiece of high energy physics activity for the foreseeable future.

\section{UNK [2]}

The UNK project would be a $3.0 \times 0.4$ $\mathrm{TeV}$ proton-proton collider with a design luminosity of $10^{33} \mathrm{~cm}^{-2} \mathrm{~s}^{-1}$. The tunnel has been excavated and $70 \%$ of the components for the (warm) $0.4 \mathrm{TeV}$ ring are completed. Twentyfive full scale superconducting magnets for the $3 \mathrm{TeV}$ ring have been tested successfully, and preparations are in progress for a preproduction run of 100 magnets. Because of the financial problems in the former Soviet Union, completing UNK by 1997-1998 as an international project is being considered.

\section{Accelerator Physics and Technology}

Talks in the parallel sessions covered some of the most important accelerator physics and technology of hadron colliders. The following are short summaries of those talks.

Superconducting Magnets $[3,4]$

There are many interrelated aspects of superconducting magnets - mechanical and quench properties, field quality, cost optimization, etc, and there are detailed articles in accelerator conferences and excellent reviews $[5,6]$ on the subject. New information about mechanical and quench properties is coming from the LHC and SSC, and talks at this conference concentrated on these.

A magnet will quench, become a normalrather than a super-conductor, at some current. Ideally that current is the "short sample" limit where the current density exceeds the critical current density. Below the short sample limit: i) quenches are caused by a sudden motion of a conductor that raises the temperature of a section of magnet above the critical temperature, ii) once a conductor has moved, it does not return to its original position, and, as a result, iii) magnets "train" - successive quenches tend to occur at higher currents. The mechanics of the magnet determine these training and quench properties. A stiff coil support won't allow conductor motion. The support should include constraints on the ends of the coil, and its stiffness can be increased by tightly clamping the magnet yoke around the collared coil assembly. This is being done in the SSC magnets which reach the short sample limit, about $10 \%$ above the design current corresponding to a field of $6.6 \mathrm{~T}$, with one or two quenches at most. The same design philosophy has been used at LBL to build a magnet operating at $1.8 \mathrm{~K}$ that reached $10.06 \mathrm{~T}$ after five training quenches. The LHC magnetic field will be somewhere between 8.5 and $10 \mathrm{~T}$. One meter long model magnets have reached over $9 \mathrm{~T}$ but have had substantial 
training. Most of the quenches are at the coil ends.

Quenches are caused by losses also. For example, the Rutherford cable used in all superconducting magnets is braided with strands crossing over each other forming loops. These crossovers have resistance; the value depends on the surface coating of the strands. When the magnet current is ramped, EMF's induced in these loops can cause currents and sufficient heating to quench the magnets if the crossovers are not resistive enough. The quench current of some prototype SSC dipoles is more than $10 \%$ below the DC value at the ramp rate of the IIigh Energy Booster. Increasing the crossover resistance should solve this particular problem.

The engineering practices to produce good superconducting magnets are understood. Some of the fundamentals of quenches, the effects of cooling, conductor size, inductance, etc, are not. They will need to be understood better as magnet performance is pushed.

\section{Cryogenics [7]}

Superconductivity is the technology that determines the energy frontier of particle physics. As a result, cryogenics is becoming increasingly important, and large cryogenic plants are part of most accelerator laboratories. There are $10-20 \mathrm{~kW}$ of cooling at $4.5 \mathrm{~K}$ and several kilometers of cold accelerator at DESY and Fermilab. The capacity at CERN will rise from $19 \mathrm{~kW}$ today to roughly $70 \mathrm{~kW}$ for LEP200 and $150 \mathrm{~kW}$ in the LHC era. The 200 $\mathrm{kW}$ capacity of the SSC will come from 10 refrigerators with $20 \mathrm{~kW}$ capacity and multiple cooling loops in a continuation of the trend towards efficient, compact cryogenic plants.

Cryogenic engineering must be integrated into a collider design. Some examples follow. Static heat loads, resistive dissipation in superconducting devices, and the impact of beam induced losses (from synchrotron radiation, for example) must be minimized. This affects parameters as basic as whether the magnet body is warm or cold and the size of the magnet bore. Heat loads have large variations in instantaneous power and duration. Quenches make extreme, localized demands for tens of seconds while cooldowns can last several days and extend over a substantial fraction of the accelerator. Energy ramps and steady operation have other load profiles. The mignets and cryogenic system must accommodate this variety. Single-phase and two-phase He have advantages and disadvantages as cooling fluids and can be used in different applications or parts of the cooling loop. For high magnetic fields He II operating below $2 \mathrm{~K}$ is feasible although the technology of cold compressors needs further development. The LHC and SSC have included cryogenics as a basic element in the optimization of cost and performance.

\section{Long Term Stability |8|}

Single particie motion in nonlinear magnetic fields is the most important accelerator physics issue in large hadron colliders. Nonlinear fields are produced by persistent currents and conductor placement errors in superconducting dipoles and quadrupoles, and nonlinear magnets are used to correct chromatic aberrations. These fields can cause unstable motion and particle losses and have the greatest effect at the injection energy where the momentum is lowest and the ratio of persistent currents to transport currents is largest. Extrapolation from the Tevatron to larger rings is uncertain - the peak energy of the Tevatron is only six times the injection energy, the nonlinearities are small due to the large bore of the Tevatron magnets, and the LHC and SSC have much larger circumferences. The recent success commissioning the HERA proton ring, where the energy ratio is twenty, removes one of those uncertainties and gives confidence in many aspects of stability analysis.

Nonlinear motion is understood in simple situations, but storage rings are complex and have many weak nonlinearities. Computer simulations are the preferred analysis technique. There are several time scales of interest. Stability for a few turns is needed to correct the 
first turn orbit and establish a circulating beam. Routine accelerator adjustments require stability for roughly $10^{4}$ turı.s, and filling both rings takes about $10^{8}$ turns. Substantial losses during that time could quench magnets. Recent studies have concentiated on the long time scale. Brute force simulations that follow particles through individual magnets for a million or more turns are possible thanks to increasingly powerful, economic computing. "Survival plots" show the aperture and losses and lead to the conclusion that beam particles are stable for long times.

The remaining question is whether the simulation models contain sufficient physics. At large amplitudes motion is irregular and not deterministic. Stochastic motion and diffusion give a better description. Diffusion has been measured in experiments at the SPS and Tevatron, but these measurements are not understood quantitatively. There are no predictions of the diffusion constants and their dependence on other parameters. The conclusions about long term stability will remain uncertain until these measurements have been explained.

\section{Injectors [9]}

The LHC and SSC will achieve high luminosities by a combination of a large number of bunches and beams with high phase space densities. Not surprisingly, this combination determines many of the parameters and specifications of the injector complex and leads to some of the most interesting accelerator physics of large colliders. Counting the linac, its ion source and preaccelerator as one, there are five accelerators in each of these complexes. For example, for the LHC those are the linac, the PS Booster, the PS, the SPS and the LHC itself.

Having a large number of bunches leads to strong coupled bunch instabilities and beam loading. Some of the instabilities are naturally damped by Landau damping; those that aren't must be cured with feedback. Beam loading shifts the optimum RF cavity frequency by amounts comparable to the revolution frequency in some of the accelerators and special RF feedback that can compensate for beam loading is necessary. This has implications i) for all the other RF controls, ii) on the installed RF power, and iii) even the conventional construction by fixing the location of the RF power sources.

The maximum phase space density is determined by the ion source, and, at best, it can be preserved through the acceleration cycle. While both designs allow for some reduction, the densities are higher than those achieved at the SPS and Tevatron. Space charge effects in the low energy boosters, the SSC LEB and the CERN PS, are a central issue and one of the dominant causes of density reduction. Space charge introduces a tune spread that is proportional to the density and $1 / \beta \gamma^{2}$ ( $\beta$ and $\gamma$ are the usual relativistic quantities: $\beta=v / c, \gamma=$ $\left.\left(1-\beta^{2}\right)^{-1 / 2}\right)$. The design tune spreads, $\Delta Q_{S C}$, are 0.35 and 0.20 in the SSC LEB and CERN PS, respectively. For comparison, at $\triangle Q_{S C}=0.35$ in the FNAL Booster there is roughly a factor of two density reduction. Such density reduction is caused by nonlinear resonances within the tune range of particles in the beam. These resonances must be compensated with magnetic nonlinearities. Experiments at the PS that included resonance compensation show that the design phase space density for the LHC can be reached, and LEB simulations lead to the same conclusion for the SSC. In addition, flexibility is being incorporated into the SSC design - the linac housing will be long enough for a future energy upgrade.

With thought about these types of issue and careful design, the LHC and SSC beams should be intense and dense enough to give the design luminosities.

\section{$\mathrm{E}^{+} \mathrm{E}^{\cdot}$ HEA VY QUARK FACTORIES}

There is a maximum charge density at the collision point caused by beam produced electromagnetic fields. This limit, called the beam-beam limit, is parametrized by $\xi$, the beam-beam tune shift. The physics of the 
beam-beam interaction is not well-understood, but there is extensive experience indicating that designing with $\xi \leqslant 0.05$ is prudent for $\mathrm{e}^{+} \mathrm{e}^{-}$ storage rings. Equation (1) for the luminosity can be written

$$
\varepsilon=\frac{N\left(\bar{x}_{c} / B\right) \xi\left(1+\sigma_{y} / \sigma_{x}\right) \gamma}{2 r e_{y}^{\beta_{y}}}
$$

when the beam-beam limit is taken into account. This equation can be evaluated for either beam provided the parameters of that beam are used throughout, and the horizontal and vertical tune shifts have been assumed to be equal. Luminosity is increased by raising $N$, the total number of particles in the beam, reducing $\beta_{y}^{*}$, and increasing, to the extent possible, the beam-beam limit.

\section{CESR [10]}

A series of upgrades including multiple bunches, low $\beta^{*}$ optics, and operating with only one interaction point have increased the integrated luminosity roughly an order of magnitude every six years. The present record performance is: $£$ (peak) $=2.5 \times 10^{32} \mathrm{~cm}^{-2} \mathrm{~s}^{-1}$, an integrated luminosity of $1.2 \mathrm{fb}^{-1}$ in 1991 , and $1.6 \mathrm{fb}^{-1}$ expected in 1992 (an average of $5 \times 10^{31} \mathrm{~cm}^{-2} \mathrm{~s}^{-1}$ for an entire year!).

Upgrades are continuing. New RF cavities and electrostatic separators will remove the present beam current limitations, and an integrated luminosity in the range 2.5 to 3.0 $\mathrm{fb}^{-1} /$ year is expected in 1993 . Starting in the beginning of 1994 the plan, called "CESR Phase II", is to make further modifications to raise currents and to use a small crossing angle to give a projected increase in the number of bunches to twenty-seven and the luminosity to $6 \times 10^{32} \mathrm{~cm}^{-2} \mathrm{~s}^{-1}$. Beyond that, superconducting cavities could be used to raise the luminosity to the $10^{33}$ range, and, if the past is a guide, the experience gained in the next few years will give additional ideas for raising the luminosity. That experience with high currents and luminosity will be valuable for future heavy quark factories also.
New Projects

There are new projects focused on each of the heavy quarks: $\Phi$-factories at Novosibirsk and Frascati, $\tau$ C-factories at the Joint Institute for Nuclear Research and in Spain, and Bfactories at Novosibirsk, KEK, Cornell, and SLAC. These projects have excellent, welldocumented particle physics justifications, and there are advanced, detailed proposals. The Frascati $\Phi$-factory is funded and under construction, but the other projects are waiting for approval or are caught up in the financial uncertainties of the former Soviet Union.

The general consensus on the way to reach high luminosity is:

1. Two rings and a large number of bunches. The luminosity depends on the total number of particles and not on the number of bunches. A large number of bunches reduces single bunch stability problems at the expense of multiple bunch instabilities, but the latter are easier to cure with feedback and RF cavity design. The interaction region tends to be simpler with a large number of bunches because they can be physically smaller without having beam-beam problems. However, the bunches must be separated close to the interaction point, and that adds complications.

2. Flat beams, $\sigma_{y}<\sigma_{x}$, because that simplifies the interaction region optics and has lower experimental backgrounds.

3. $\beta_{y}^{*} \leq 1-3 \mathrm{~cm}$ limited by the length of the bunch and momentum dependence of the interaction region focusing if it is made stronger to reduce $\beta_{y}^{*}$ further.

Only the Novosibirsk $\Phi$-factory departs substantively from this consensus. The total currents are huge, 1 - 2 A as compared to $\sim 100$ $\mathrm{mA}$ in CESR, and heavy demands are placed on the RF and vacuum systems.

Having made these basic choices, work is concentrated on refinements and developing critical components. With the large number of projects and extensive activity it is possible to give only a flavor of the progress. Individual contributions to the conference should be referred to for details [11-15]. 
The experimental detector and storage ring interaction region are intimately related. They are competing for the same space and influence each other strongly. Experimental backgrounds are particularly important, and there have been extensive simulations of the backgrounds from beam particles degraded by gas scattering and from synchrotron radiation. These simulation have led to major design choices such as the use of flat beams and determine some of the accelerator parameters. They lead to the conclusion that the backgrounds can be reduced sufficiently, also. One simulation has been checked at Cornell |16|. Currents in tracking chambers and trigger rates were compared with Monte Carlo results and found to be in excellent agreement. This shows that backgrounds can be understood, provides a "standard" simulation, and builds confidence in the conclusion of acceptable background levels.

Integration of accelerator and detector components (focusing quadrupoles, compensating solenoids, vacuum pumps, synchrotron radiation masks, vertex detectors, thin beam pipes, and all of the associated fluids and cables) is a major engineering job. B- and $\tau$ C-factories have to accommodate a small radius interaction region beam pipe for vertex detectors, but the $\Phi$-factories have a new and unusual problem. A large radius pipe is needed for a $K^{0}$ decay volume. The focusing quadrupoles must have a small radius for adequate strength, and the transition to the decay region creates an RF cavity-like structure that can extract energy from the beam and lead to heating and interesting beam dynamics.

Beam bunches interact through electromagnetic fields with decaly times greater than the time between bunches. Multiple bunch instabilities can be prevented by reducing the Q's, the quality factors, of unwanted resonances. When the Q's cannot be reduced sufficiently, instabilities must be controlled with feedback. High Q resonances can be avoided in many structures, but that is impossible in an RF cavity. However, the Q's for all but the accelerating mode (the fundamental mode) can be reduced by the following. First, only one or two RF cells are combined into a single cavity. That avoids complicated spatial structures of the unwanted higher modes. It has the added benefit of minimizing the fundamental mode power passing through each RF window separating the cavity from the power source. Second, waveguides that are cut-off for the accelerating mode, but not for the higher frequency modes, are placed appropriately to couple strongly to the higher modes and remove energy from them. It has been demonstrated that the Q's of higher modes of room temperattire cavities can be reduced sufficiently, to 100 or less, without affecting the fundamental mode.

The same ideas, one or two cells per cavity and strong coupling to higher modes, work for superconducting cavities also. Superconducting cavitics have high accelerating gradients, and that has the advantage of reducing the number of cavities. Those gradients can be reached even with a large opening for the beam. With a large opening there are only a few high $Q$ resonant modes and the beam pipe itself becomes the higher mode waveguide. RF absorbers placed in a room lemperature section of the beam pipe absorb higher mode energy. The potential advantages of superconducting RF are balanced by uncertainties associated with transferring large RF power to the beams. This is a new mode of operation that will be tested at Cornell in late 1993.

Typical bunch spacings are $1-3 \mathrm{~m}$, and feedback systems must detect and correct longitudinal and transverse errors of such closely spaced bunches. This requires i) high frequency detectors, ii) high frequency, high power kickers, and iii) signal processing that can keep track of a large number of bunches. Digital Signal Processing has the bandwidth and data handling capabilities, and it forms the basis of the SI.AC/LBL/LLNL feedback system. This feedback has attracted interest at the Advanced Light Source, and a prototype is under construction for testing there. 
Conclusions

There is general agreement on the route to high luminosity, and critical elements are being designed, engineered, and tested. The examples abc ve were chosen to give a flavour of these developments. These colliders should reach the design luminosity, and heavy quark factories are sure to be a part of the future of high energy physics.

\section{HIGH ENERGY E $\mathrm{E}^{+}$COLLIDERS}

\section{TRISTAN |12].}

TRISTAN is zunning at $\mathrm{E}_{\mathrm{CM}}=58 \mathrm{GeV}$, and the luminosity has exceeded $1 \mathrm{pb}^{-1} / \mathrm{day}$. The goal is to accumulate $300 \mathrm{pb}^{-1}$ total luminosity. It is anticipated that this will be achieved in 1994. Extensive experience has been gained with superconducting $R F$ in a working accelerator. The $48 \mathrm{~m}$ of superconducting cavities has operated for several years with an average gradient of 4.7 $\mathrm{MV} / \mathrm{m}$.

\section{LEP [17]}

A new magnetic configuration compatible with i) $\mathrm{E}_{\mathrm{CM}}=\mathrm{m}_{\mathrm{Z}}$, ii) $\mathrm{E}_{\mathrm{CM}}>\mathrm{W}$-pair threshold, iii) transverse polarization, and iv) electrostatically separated orbits was commissioned at the beginning of the 1992 LEP run. This was a major change, but the initial difficulties have been solved. At the time of this conference LEP was running with $\mathfrak{f}=$ $10^{31} \mathrm{~cm}^{-2} \mathrm{~s}^{-1}$ and had an integrated luminosity, over $8 \mathrm{pb}^{-1}$, exceeding that of the 1991 run at the comparable time.

The beam-beam tune shift and single bunch current are above their design values, and the luminosity and total current are approaching theirs. The total current limit is thought to be caused by the beam-beam interaction at the injection energy where the beams are electrostatically separuted but there are residual effects. With the solution of this problem LEP should exceed all of its design goals.
The new magnetic configuration is the foundation for future LEP improvements. The luminosity can be doubled by increasing the number of bunches from four to eight with separated orbits. This mode of operation has been tested successfully, and it should become routine by the end of September of this year. The energy calibration can be improved by resonant depolarization in the same configuration used for collisions.

The new magnet configuration is part of LEP2, the energy increase to above the W-pair threshold. Superconducting RF is the heart of LEP2. At 90 GeV/beam the energy loss per turn is roughly $2.2 \mathrm{GeV}$. This energy loss must be made up by the RF system. The LEP2 RF system will have 192 superconducting cavities with a gradient of about $5 \mathrm{MV} / \mathrm{m}$ together with the 120 existing copper cavities. About $15 \%$ of the superconducting cavities have been delivered and accepted, and the full complement is expected in 1994. The physical plant and atilities must be increased substantially also - new RF power sources, cryogenics, water cooling, power supplies, etc. This work is on target for running in 1994 at $\mathrm{E}_{\mathrm{CM}}=180 \mathrm{GeV}$.

$S L C[18]$

1992 has been a critical year for the SLC and for future linear coliiders. Despite its peculiarities the SLC is the prototype linear collider, and the feasibility of performing high energy physics experiments at linear colliders must be judged based on the SLC experience. Luminosity, uptime, backgrounds, and polarization are all important and must be achieved simultaneously. The 1992 SLC run has shown that this is possible with the best evidence being the SLD results presented at this conference.

The present SLC performance is: $\mathfrak{i}$ $2 \times 10^{29} \mathrm{~cm}^{-2} \mathrm{~s}^{-1}$ with peaks up to $2.5 \times 10^{29} \mathrm{~cm}^{-2} \mathrm{~s}^{-1}$, uptime $\sim 60-70 \%$, sustained running with backgrounds sufficiently low for SLD data taking, and a polarization $22-24 \%$ at the interaction point. A large number of 
techniques had to be developed to reach this level of periormance. This work is documented in the accelerator conference proceedings and has been summarized by J. Seeman [19]. The recent progress on polarization and feedback are highlighted here.

Producing polarization at the interaction point requires sophisticated physics and technology throughout the accelerator, but the most difficult aspect has proven to be the polarized source itself. There state-of-the-art vacuum, high voltage, and materials engineering combine with incompletely understood physics of quantum efficiency, polarization, and intensity saturation to determine performance. One source meeting the SLC specifications has been operating routinely and reliably since April 1992. The cathode is bulk GaAs with $27 \%$ polarization. Depolarization during acceleration, damping, and transport reduce this to $22-24 \%$ at the interaction point. There are a number of options for increasing the polarization by modifying the cathode: cooling of bulk GaAs to $150 \mathrm{~K}$, changing the cathode to AlGaAs, using thin GaAs and a different wavelength excitation, and using a strained GaAs crystal [20]. The first three are short term (intended for the 1993 run) and promise polarization at the cathode in the $40-45 \%$ range. Some are being tested now. An operational source using a strained GaAs crystal is still under development and is at least a year away. It would have $85 \%$ polarization at the cathode.

Beam properties (orbits for example) vary from pulse-to-pulse and drift. Pulse-to-pulse jitter must be reduced to acceptable levels by stabilizing accelerator components or special beam dynamics like BNS damping [21]. Feedback to control drifts has always been part of the SLC, but recently there has been an innovation of using database driven feedback system based on digital control theory [22]. These feedbacks sample the beam at $20 \mathrm{~Hz}$, correct noise below $2 \mathrm{~Hz}$, and respond to step changes in $0.2 \mathrm{sec}$. These loops are adaptive: they recognize and compensate for changes in the accelerator, and they are cascaded: they communicate with each other to insure that only one loop corrects a particular error. New loops are implemented with database modifications and modest hardware installation. Feedback has become the preferred solution to many problems, and 22 loops, about four times the number initially planned, are active currently. They are a major contributor to the increased luminosity, uptime and efficiency of the SLC, and this development is important for future linear colliders.

Coniinuing work of the type illustrated will increase the polarization and luminosity of the SLC, and it will lead to many SLD results at future conferences. The experience gained will be invaluable for the future through the understanding of the practicalities of operating a linear collider for particle physics.

\section{Future Linear Colliders}

Some of the principal parameters for linear colliders with $\left.\mathrm{E}_{\mathrm{CM}}=5()\right) \mathrm{GeV}$ from the 1992 ECFA workshop on $e^{+} e^{-}$linear colliders (LC92) held a week before Dallas are given in Table 1 [23]. There is striking diversity in these parameters representing different judgements about various factors.

The ease of improving technology . The DESY/Darmstadt and lowest frequency JLC are the most conservative in this regard. They take advantage of over forly years of experience with S-band $(\sim 3 \mathrm{GHz}) \mathrm{RF}$. Some new components, high power klystrons in particular, are needed. The NLC and the other versions of the JLC extend the basis of present day linacs - high peak power klystrons and modulators - to higher frequencies. Klystrons and accelerator structures must be developed for those frequencies. TESLA relies on substantial improvements in the cost and accelerating gradient of superconducting $R F$, but it has the advantage of a substantially larger collision spot. VLEPP requires a number of innovations in alignment, jitter reduction, collimation, and beam dynamics to obtain high luminosity with a single intense bunch. CLIC uses a high RF frequency that leads to stringent alignment and 
Table 1. Selected Preliminary Linear Collider Parameters for $\mathrm{E}_{\mathrm{CM}}=500 \mathrm{GeV}^{*}$ [23]

\begin{tabular}{lcccccccc}
\hline \hline & $\begin{array}{l}\text { RF Freq } \\
(\mathrm{GHz})\end{array}$ & $\begin{array}{l}\text { Gradient } \\
(\mathrm{MeV} / \mathrm{m})\end{array}$ & $\begin{array}{l}\text { Rep Rate } \\
(\mathrm{Hz})\end{array}$ & $\begin{array}{l}\text { Bunches/ } \\
\text { RF Pulse }\end{array}$ & $\begin{array}{l}\sigma_{\mathrm{x}} / \sigma_{\mathrm{y}} \\
(\mathrm{nm})\end{array}$ & $\begin{array}{l}\mathrm{P}_{\mathrm{B}} \\
(\mathrm{MW})\end{array}$ & $\begin{array}{c}\mathrm{T} \\
\left(10^{33} \mathrm{~cm}^{-2} \mathrm{~s}^{-1}\right)\end{array}$ \\
\hline TESLA & 1.3 & 25 & 10 & 800 & $640 / 100$ & 16.5 & 0.035 & 8 \\
DESY/Darmstadt & 3.0 & 21 & 50 & 172 & $400 / 32$ & 7.5 & 0.058 & 4 \\
NLC (SLAC) & 11.4 & 50 & 180 & 90 & $300 / 3$ & 4.2 & 0.10 & 9 \\
& 2.8 & 22 & 50 & 55 & $300 / 3$ & 1.6 & 0.20 & 4 \\
JLC (KEK) & 5.7 & 40 & 100 & 90 & $260 / 3$ & 3.6 & 0.20 & 7 \\
& 11.4 & 40 & 150 & 90 & $260 / 3$ & 3.8 & 0.15 & 6 \\
VLEPP & 14.0 & 108 & 300 & 1 & $2000 / 4$ & 2.4 & 0.06 & 12 \\
CLIC (CERN) & 30.0 & 80 & 1700 & $1-4$ & $90 / 8$ & $.4-1.6$ & 0.15 & $1-3$ \\
\hline \hline
\end{tabular}

* $\sigma_{\mathrm{x}}$ and $\sigma_{\mathrm{y}}$ are the horizontal and vertical beam sizes, $\mathrm{P}_{\mathrm{B}}$ is the power of one beam, and $\mathrm{T}$ is given by eq. (4).

TESLA uses superconducting RF; all others use room temperature RF.

fabrication specifications and significant beam dynamics problems. An elegant "two-beam" RF power source replaces conventional klystrons and requires substantial development.

Costs - New technologies promise significant, but uncertain, cost reductions. Older technologies have better established costs, but these tend to be high and must be lowered through engineering and mass production. The experience of the SSC, an accelerator based on mature technology and a detailed design, teaches us that present day linear collider cost estimates should not be taken seriously.

Extension to higher energies - For room temperature RF, high gradients and high RF frequency tend to be better for high energies. They reduce the accelerator length and improve the energy efficiency, respectively. The NLC, high frequency JLC's, and VLEPP are optimized for $\mathrm{E}_{\mathrm{CM}}=500 \mathrm{GeV}-1 \mathrm{TeV}$. The $\mathrm{S}$ band colliders would be straining to go beyond $500 \mathrm{GeV}$, and the CLIC parameters are those of a multi-TeV collider scaled down to $\mathrm{E}_{\mathrm{CM}}=500$ $\mathrm{GeV}$ for purposes of comparison. The energy reach , $f$ TESLA depends on how close the fundamental gradient limit of $\sim 50 \mathrm{MeV} / \mathrm{m}$ in $\mathrm{Nb}$ can be approached.

Experimental backgrounds and energy spectrum - A large number of photons are radiated in the intense electromagnetic fields at the collision point. These "beamstrahlung" photons degrade the energy spectrum of the primary $e^{+} e^{-}$collisions and produce experimental backgrounds directly and through $\mathrm{e}^{+} \mathrm{e}^{-}$pairs and hadrons from $\gamma \gamma$ collisions. These effects are parametrized by $\Upsilon=\gamma B / B_{C}$ where $\mathrm{B}$ is the effective electromagnetic field strength and $B_{C}=4.4 \times 10^{13} \mathrm{G}$ is the Schwinger critical field. In terms of beam properties an approximate expression for $\Upsilon$ is

$$
\mathrm{r}=\frac{\mathrm{r}_{\mathrm{e}}^{2}}{\alpha} \frac{\gamma_{\mathrm{n}}}{\sigma_{\mathrm{L}}\left(\sigma_{\mathrm{x}}+\sigma_{\mathrm{y}}\right)}
$$

where $\mathrm{n}$ is the number of particles per bunch, $\sigma_{\mathrm{L}}$ is the bunch length, and $r_{\mathrm{e}}$ and $\alpha$ are the classicai radius of the electron and the fine structure constant, respectively. All designs use flat beams to minimize beamstrahlung. Most of the particles produced in electromagnetic processes are in the forward direction and should not cause backgrounds with a properly designed interaction region. Relatively rare $\mathrm{e}^{+} \mathrm{e}^{-}$ pairs with $\mathrm{P}_{\text {TRANSVERSE }} \geq 20 \mathrm{MeV} / \mathrm{c}$ are an important background, and there was speculation that the "minijet", $\gamma \gamma \rightarrow$ hadrons, cross section was large [24]. Preliminary HERA measurements of the total $\gamma$ p cross section indicate that this is not the case $[25,26]$.

Discussions have concentrated on backgrounds originating from collisions 
themselves. Beam halos are likely to be as serious and providing proper collimation will have a strong influence as designs evolve.

Tolerances and beam power - While there are considerable differences in estimates of the tolerable amount of beamstrahlung, $\Upsilon$ will be determined by experimental backgrounds. The luminosity can be written in terms of $\Upsilon$

$$
\varepsilon=\frac{1}{4 \pi} \frac{\alpha}{r_{e}^{2} m^{2}} \frac{r \sigma_{L}}{\gamma^{2}} \frac{{ }_{B}}{\sigma_{y}} .
$$

Disruption, focusing during the collision, has been neglected. The free parameters are $\sigma_{\mathrm{L}}, \sigma_{\mathrm{y}}$, and $\mathrm{P}_{\mathrm{B}}$. Since a bunch can occupy only a small fraction of an $R F$ wavelength, $\sigma_{L}$ is one of the many factors affecting the choice of $\mathrm{RF}$ frequency. That leaves a tradeoff between beam power and spot size. Effectively, this is a tradeoff between tolerances and beam power.

Bright beams and a final focus system with low order aberrations corrected are required for small spots. Beam brightness depends on the damping rings producing the beams and beam generated fields experienced during acceleration. Damping ring and main accelerator tolerances become more stringent as the brightness increases. The main accelerator tolerances depend strongly on the RF frequency with low frequency favored. That adds tolerances to considerations when selecting the RF frequency. Correcting optical aberrations in the final focus leads to tight alignment, stability, and field quality tolerances there, also. Increasing the beam power relaxes tolerances, but there are limits to beam power. There must be efficient transfer of energy to the beam, and beam handling, collimation and accelerator protection, become difficult as power increases.

Table 1 and the paragraphs following it show the interconnected considerations entering into the most basic paramete"s and the diversity of opinion about the best design. How will the thoices be narrowed and the best approach decided? Four important factors will be continued experience with collisions at the SLC, SLC beam dynamics experiments, prototypes of critical components, and prototypes of systems.
Experiments studying beam generated fields, acceleration of that beams and multiple bunches, advanced orbit correction techniques, and electrodynamics in the strong field regime of beamstrahlung are planned at the SLC. A new facility, the Final focus Test Beam, is nearing completion. It is a combination of a beam dynamics experiment and a system prototype. Optics with the appropriate corrections will be tested in this prototype final focus. Demagnifications even greater than those planned in many of the designs should be reached.

All of the designs have critical components. Highlights of progress on some of these follows. Multiple bunch acceleration depends on the decoherence or the damping of beam generated fields leading to intrabunch interactions. Decoherence is accomplished by spreading the resonant frequencies of unwanted modes while damping requires reduction of their Q's. Both methods work, and the issue is reducing complexity to lower manufacturing costs. A radial transmission line with an RF choke for the fundamental mode allows damping of higher modes without affecting the fundamental and has the advantage of being a rotationally symmetric structure that could be turned on a lathe 1271. A model cavity has been constructed and shown to have the predicted RF properties.

The gradient of superconducting cavities is limited by field emission. Field emitted electrons extract energy from the cavity fields and generate heat when they strike the wall. Processing the cavities with short burst of high power RF burns up the field emitters which are usually imperfections introduced during manufacturing. Cavities processed in this way have reached gradients in the range $17-27$ $\mathrm{MV} / \mathrm{m}[28]$.

$\mathrm{X}$-band (11.4 Gllz) colliders need klystrons capable of generating $50 \mathrm{MW}$ of $\mathrm{RF}$ power for $1 \mu \mathrm{s}$. Klystrons with this power combined with RF pulse compression give a gradient of about 5() $\mathrm{M} \approx \mathrm{V} / \mathrm{m}$ with a klystron for every 3-4 $\mathrm{m}$ of accelerator. A klystron with that performance has been tested suc essfully at 
SLAC [29]. While the efficiency must be improved, it is the first demonstration of an Xband RF power source.

Prototype systems to study both beam dynamics and system engineering are planned or under construction. These include:

1. A $500 \mathrm{MeV}$ TESLA prototype to be constructed at DESY. The aims are to demonstrate a gradient of $15 \mathrm{MV} / \mathrm{m}$, to meet cost goals, and to test a high gradient superconducting linac with beam.

2. A $450 \mathrm{MeV}$ prototype of the DESY/Darmstadt collider that will test long pulse, high power, multiple bunch operation of an S-band linac.

3. The Accelerator Test Facility at KEK combines a $1.5 \mathrm{GeV}, \mathrm{S}$-band linac with a prototype damping ring. The damping ring will produce beams with brightness, single bunch charge, and bunch train structure covering many of the colliders in Table 1 . New levels of tolerances, control of beam generated fields, extraction kicker stability, etc will be reached in accomplishing this.

4. Interaction region optics will be studied at the Final Focus Test Beam discussed above.

5. The NLC Test Accelerator planned at SLAC will be a $540 \mathrm{MeV} X$-band linac based on prototype klystrons and accelerator structures. The primary goal is to construct and reliably operate an engineered section of the NLC linac. Beams dynamics in an X-band linac will be studied also.

6. One of the most challenging aspects of CLIC is the two-beam RF power source. A low energy beam with bunchlets spaced at $\lambda=1 \mathrm{~cm}$ must be created, accelerated, and energy extracted from it. A beam with this structure will be generated by an RF gun at the CLIC Test Facility. The beam will then be accelerated and energy extraction demonstrated.

These prototypes will narrow the options in Table 1. The widely accepted goal of proponents is a proposal for a high energy linear collider by the mid 1990's.

\section{REFERENCES}

1. V. K. Bharadwaj, ICHEP session PA-22a.

2. L. P. Soloviev, ICHEP session PA-23b.

3. A. Devred, ICHEP session PA-22a.

4. R. Palmer, ICHEP session PA-23a.

5. R. Palmer and A. V. Tollestrup, Ann Rev Nucl Part Sci 34, 247 (1984).

6. K.-H MeB and P. Schmüser, CERN Accel School - Supercond in Part Accel, 87 (1989).

7. Ph. Lebrun, ICHEP session PA-23a.

8. R. Talman, ICHEP session PA-22a.

9. W. T. Weng, ICHEP session PA-22a.

10. D. L. Hartill, ICHEP session PA-22b.

11. D. Rice, ICHEP session PA-23b.

12. K. Satoh, ICHEP session PA-22b.

13. T. Fieguth, ICHEP session PA-22b.

14. G. Vignola, ICHEP session PA-22b.

15. A. Sissakian, ICHEP session PA-22b.

16. S. Henderson, ICHEP session PA-23b.

17. S. Myers, ICHEP session PA-22c.

18. N. Phinney, ICHEP session PA-22c.

19. J. T. Seeman, Ann Rev Nucl Part Sci 41, 389 (1991).

20. T. Maruyama et al, Phys Rev Lett 66, 2376 (1991).

21. V. E. Balakin, A. V. Novokhatsky and V. P. Smirnov, Proc of 12 th Int Conf on HighEnergy Accel, 119 (1983).

22. F. Rouse et al, SLAC-PUB-5681 (1991).

23. G. Loew, Summary Talk 1992 ECFA Workshop on $\mathrm{e}^{+} \mathrm{e}^{-}$Linear Colliders.

24. M. Drees and R. M. Godbole, Phys Rev Lett 67, 1189(1991).

25. B. Löhr, ICHEP session PA-24.

26. F. Eisele, ICHEP session PA-24.

27. T. Shintake, KEK Preprint 92-51 (July, 1992).

28. H. Padamsee, ICHEP session PÁ-23a.

29. G. Caryotakis, 1992 ECFA Workshop on $\mathrm{e}^{+} \mathrm{e}^{-}$Linear Colliders.

\section{DISCLAIMER}

This report was prepared as an account of work sponsored by an agency of the United States Government. Neither the United States Government nor any agency thereof, nor any of their employees, makes any warranty, express or implied, or assumes any legal liability or responsibility for the accuracy, completeness, or usefulness of any information, apparatus, product, or process disclosed, or represents that its use would not infringe privately owned rights. Reference herein to any specific commercial product, process, or service by trade name, trademark, manufacturer, or otherwise does not necessarily constitute or imply its endorsement, recommendation, or favoring hy the United States Government or any agency thereof. The views and opinions of authors expressed herein do not necessarily state or reflect those of the United States Government or any agency thereof. 

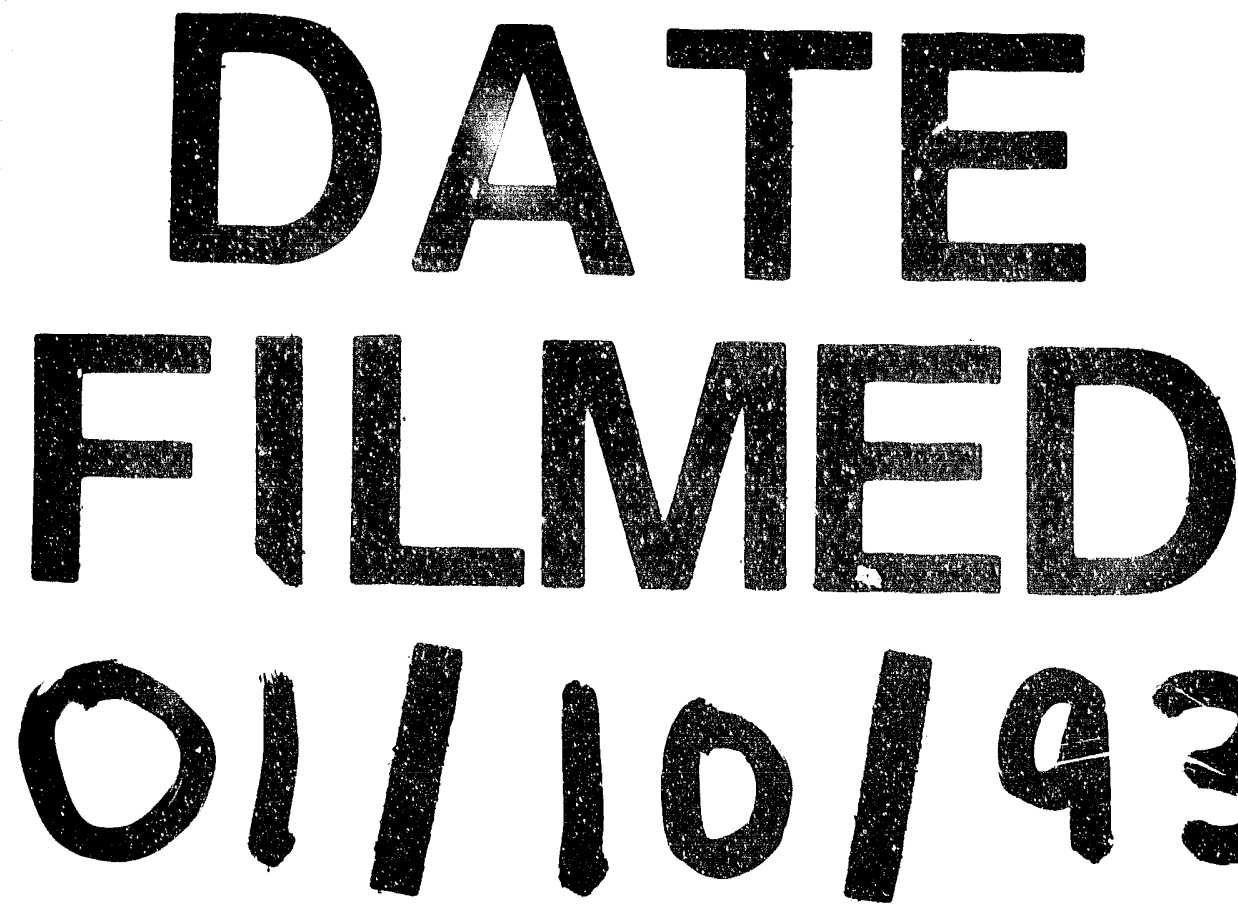

93 